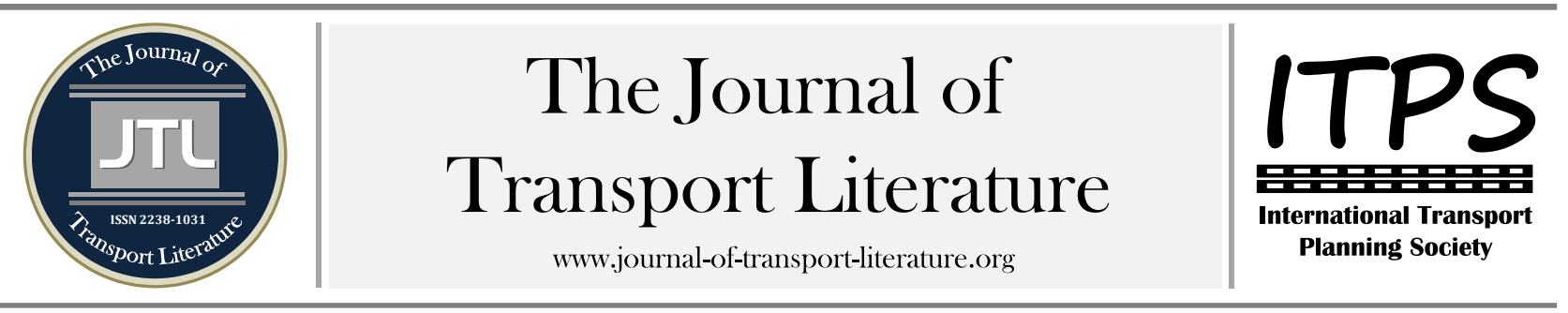

\title{
Localização e concentração de atividades logísticas no Brasil
}

\author{
André Catapan; Mônica Maria Mendes Luna ${ }^{+}$
}

Universidade Federal de Santa Catarina, Florianópolis, Brasil

\section{Article Info}

Palavras-chave:

Instalações Logísticas

Zonas Logísticas

Plataformas Logísticas

Decisão de localização

Submitted 19 Jun 2015;

received in revised form $5 \mathrm{Jul} 2015$

accepted 12 Jul 2015.

Licensed under

Creative Common

CC-BY 3.0 BR.

\section{Resumo}

O presente trabalho analisa a localização e concentração das atividades logísticas no Brasil, com destaque para os estados de Pernambuco, Goiás e Santa Catarina. Para identificar estas concentrações foram usados dados relativos ao número de funcionários que atuam nos vários grupos de atividades econômicas relacionados a transporte e logística. Os resultados mostram que há concentrações de atividades logísticas em torno dos grandes mercados consumidores, dos complexos industriais, de algumas zonas de produção agrícola e dos terminais portuários. Com base na revisão de literatura, a caracterização dos fatores que contribuem para a decisão de localização destas instalações, bem como para o fenômeno de concentração, também é apresentada. Por fim, as concentrações de atividades logísticas são classificadas de acordo com a taxonomia proposta por Savy (2005).

+ Corresponding author. Universidade Federal de Santa Catarina, Cenrtro de Tecnologia. Departamento de Engenharia de Produção e Sistemas. Campus Universitário. 88040900 - Florianópolis, SC - Brasil.

E-mail address: monica.luna@ufsc.br.

\section{Introdução}

A infraestrutura de transportes de uma região tem importante papel no seu desenvolvimento, visto que constitui condição básica para a realização de trocas econômicas entre locais espacialmente dispersos. Essa infraestrutura não diz respeito apenas às vias, mas também a uma miríade de instalações logísticas, as quais dão suporte a operações comerciais e industriais, facilitando o fluxo de bens e informações através de fronteiras locais, regionais, nacionais e internacionais.

A localização dessas instalações sofre influência de inúmeros fatores e constitui uma decisão que afeta o desempenho das empresas e, ao mesmo tempo, interessa ao poder público, uma vez que a concentração espacial das atividades logísticas pode promover o desenvolvimento de uma região, mas também trazer consequências indesejadas, como aquelas decorrentes de um fluxo intenso de veículos de cargas. Avaliar os fatores que influenciam a decisão de localização das instalações logísticas, bem como o fenômeno de concentração destas instalações, pode fornecer subsídios para decisões concernentes ao planejamento e organização do território.

0 presente trabalho apresenta uma revisão da literatura sobre este tema (seção 1) e identifica as principais concentrações de atividades logísticas no Brasil, bem como os fatores que mais contribuem para esta tendência (seção 2). Considerando os estados que se destacaram pelo elevado crescimento de atividades logísticas nos últimos anos, o estudo, na seção que trata de análise e discussão dos resultados (seção 3), mostra que estas instalações se concentram em torno dos grandes mercados consumidores, de complexos industriais, de importantes zonas de produção agrícola e das zonas portuárias. Além disso, o trabalho classifica estas concentrações de atividades logísticas de acordo com a taxonomia proposta por Savy (2005), nesta mesma seção. Por fim, são apresentadas as conclusões.

\section{As instalações logísticas: decisão de localização e o fenômeno da concentração}

As instalações logísticas têm se tornado elementos determinantes do desempenho das cadeias de suprimentos ao longo das últimas décadas. Embora a localização destas instalações seja uma decisão de cada organização visando, principalmente, reduzir custos de transporte, observa-se, segundo Pipame (2009 apud Masson e Petiot, 2010), que a definição de áreas destinadas a estas instalações passou a ser vista também como instrumento de desenvolvimento e da organização do território. Isto se deve, em parte, às consequências do fenômeno da aglomeração de atividades logísticas, a qual permite ganhos significativos de escala e outras economias de localização, mas gera, ao mesmo tempo, problemas ambientais e externalidades negativas que devem ser consideradas (Masson e Petiot 2010; Masson e Petiot 2012; Savy 2005).

0 problema da localização de indústrias é tratado há muito tempo na literatura, podendo-se destacar as teorias apresentadas por Von Thunen, Weber e Losch (Ballou 2001; Masson e Petiot 2010). Dentre estes autores, a obra "Uber den Standort der Industrien", de Alfred Weber em 1909, deu início à moderna teoria da localização industrial. 0 autor identificou três fatores primordiais para a definição da localização de uma indústria: os custos de transporte, os custos relacionados à mão de obra e, por fim, o mercado.

No que diz respeito às instalações logísticas, Masson e Petiot $(2010 ; 2012)$ afirmam que a lógica da localização dessas instalações se situa entre aquela da indústria e dos serviços. Assim, dentre os fatores que relacionam estas instalações às industriais estão a necessidade de espaços, a relevância dos custos de transporte, a disponibilidade de fatores de produção e 
as externalidades negativas geradas. 0 acesso ao mercado, por sua vez, está associado à lógica de serviços. Ainda em relação à localização das instalações, Rodrigue et al (2013) identifica três níveis distintos de decisão: i) o micro, que diz respeito à própria instalação, tratando de fatores como a oferta de terreno, a visibilidade e a natureza deste e os acessos a transporte; ii) o meso, que inclui aspectos relativos à disponibilidade de mão de obra, materiais, energia e proximidade de fornecedores e clientes; e iii) o macro, que trata do ambiente socioeconômico e leva em consideração o capital, a oferta de subsídios, as regulamentações, os impostos e a tecnologia. A importância de cada fator varia conforme a natureza da atividade e, embora não seja uma regra, a localização de atividades econômicas semelhantes se baseia em critérios semelhantes. Assim, atividades primárias tomam por base, principalmente, a disponibilidade dos recursos naturais e da capacidade de transportá-los; o setor secundário, por sua vez, leva em conta fatores como custos de mão de obra, disponibilidade de energia e proximidade dos fornecedores e consumidores; atividades terciárias, orientadas à venda, localizam-se, normalmente, próximas aos principais mercados consumidores (Rodrigue et al, 2013).

A presença de vários fatores em uma determinada região promove o fenômeno da concentração das atividades logísticas. Masson e Petiot (2012) explicam que as atividades logísticas, como armazenagem e transporte, necessitam cada vez mais de investimentos em infraestrutura, equipamentos e tecnologias e, a concentração destas atividades pode permitir o compartilhamento de ativos e a consolidação de fluxos, ou seja, a racionalização dos custos logísticos. Há ainda externalidades locais, como aquelas associadas à especialização da mão-de-obra.

No que diz respeito às pesquisas sobre as concentrações de instalações logísticas pode-se destacar, além dos trabalhos de Masson e Petiot (2010; 2012), as pesquisas de Savy (2005) e Jurásková e Macurová (2013). Este último analisa os fatores que explicam a tendência de concentração de atividades logísticos na República Tcheca. 0 trabalho de Savy (2005), por sua vez, propõe uma taxonomia para a concentração espacial de atividades logísticas e distingue quatro tipos: i) o estabelecimento logístico, que pode ser um entreposto, um centro de triagem, um armazém de uma indústria ou distribuidor; ii) um espaço com instalações especializada em logística, denominada genericamente de zona, ou plataforma se for formal ; iii) um polo logístico que compreende diversas zonas logísticas em um determinado perímetro e; iv) uma área logística, que corresponde a um agrupamento numa escala de metrópole ou região.

A maior parte dos trabalhos que abordam estas concentrações logísticas está limitada, todavia, às plataformas: estudos que analisam a importância destas instalações na cadeia de suprimentos (Cambra-Fierro e Ruiz-Benitez 2009; Jařemskis e Vasiliauskas 2007; Krzyzaniak, Hajdul, e Fechner 2012; Rodrigue e Notteboom 2009); o impacto sobre o desenvolvimento de determinada região com a criação de plataformas (Afandizadeh e Moayedfar 2008; Jaržemskis 2007; Li 2011; Tambi et al. 2013); a problemática da governança das plataformas (Silva, Senna, Senna, \& Júnior, 2013) ou análise dos diferentes termos utilizados para designar as plataformas logísticas (Grundey e Rimienė 2007; Higgins, Ferguson, e Kanaroglou 2012; Meidutè 2005; Silva et al. 2014).

\section{Procedimentos Metodológicos}

A presente pesquisa pode ser considerada, em relação a seus objetivos, como descritiva e utiliza tanto dados quantitativos quanto qualitativos. No que se refere aos procedimentos técnicos, foram utilizadas a pesquisa bibliográfica e documental. Com o objetivo de identificar concentrações de instalações logísticas no Brasil, tomou-se por base o número de empregados do setor de logística e transporte nos diversos municípios brasileiros. Numa primeira etapa foram identificadas as atividades econômicas relacionadas à prestação de serviços logísticos, segundo a Classificação Nacional de Atividades Econômicas (CNAE 2.0) do IBGE. A partir desta definição, obteve-se o número de postos de trabalho desses grupos de atividades, oriundos da Relação Anual de Informações Sociais (RAIS) do Ministério do Trabalho e Emprego (MTE), em todos os municípios brasileiros entre os anos de 2006 a 2013. Os dados foram obtidos na plataforma DARDO do MTE e, em seguida, tratados com o software Microsoft Excel. Os 5570 municípios foram, então, codificados de acordo com a base de municípios do IBGE de 2014 e, posteriormente, os dados referentes a estes incluídos no software G4Decision, o qual permite a visualização espacial animada e interativa dos mesmos. Dados relativos ao PIB de cada unidade federativa e à população dos municípios analisados também foram utilizados visando subsidiar as análises conduzidas no presente trabalho.

Após identificados os 10 estados com maior concentração de atividades logísticas no ano de 2013, identificou-se as taxas médias de crescimento de todos estes entre os anos de 2006 a 2013 e comparou-se com a média nacional no mesmo período. Fatores que contribuem para a concentração das atividades logísticas, em especial aqueles listados nos trabalhos de Masson e Petiot (2010; 2012), foram identificados nos estados de Pernambuco, Goiás e Santa Catarina, os quais apresentaram as maiores taxas no período analisado.

\subsection{As atividades econômicas e o setor de logística e transporte}

Dentre as várias atividades de logística e transporte, listadas na CNAE 2.0, foram consideradas, com o objetivo de identificar concentrações de atividades logísticas, as divisões e grupos apresentados no quadro 1.

\begin{tabular}{|ll|}
\hline \multicolumn{1}{|c|}{ Divisão } & \multicolumn{1}{c}{ Grupo } \\
\hline Transporte Terrestre & Transporte ferroviário e metroferroviário de cargas \\
& Transporte rodoviário de cargas \\
Transporte Aquaviário & Transporte dutoviário \\
& Transporte Marítimo de cabotagem e longo curso \\
Transporte Aéreo & Transporte por navegação interior \\
Armazenamento e atividades auxiliares dos transportes & Transporte aéreo de carga \\
& Armazenamento, carga e descarga \\
Correio e outras atividades de entrega & Atividades relacionadas à organização do transporte de carga \\
& Atividades de correio \\
& Atividades de malote e de entrega \\
\hline
\end{tabular}

\section{Quadro 1 - Atividades relacionadas aos setores de transporte e logística}

Fonte: Elaboração própria com base nos dados da CNAE 2.0.

Com base nos dados obtidos e tratados usando o software G4Decision, observa-se uma evolução na concentração do número de empregados no setor de transporte e logística no Brasil entre os anos de 2006 e 2013, período no qual o número absoluto de postos de trabalhos em todas as divisões analisadas teve crescimento de $65,32 \%$, com taxas médias anuais próximas a 7.5\%. A figura 1 mostra as concentrações destas atividades em algumas áreas. 


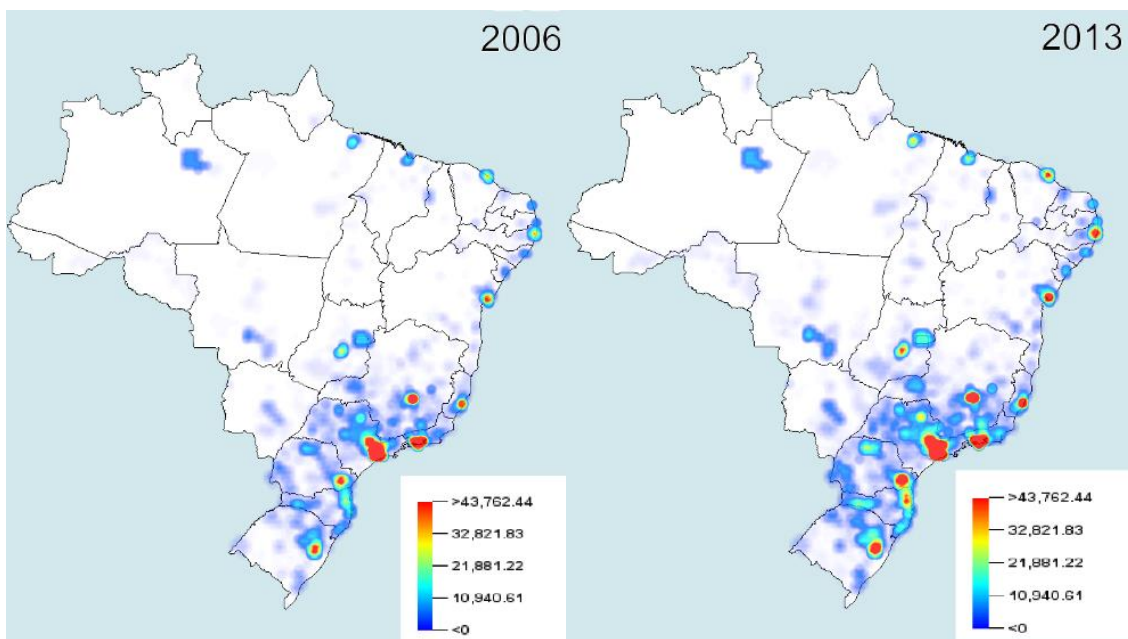

Figura 1 - Evolução do número de empregados nas atividades de logística e transporte 2006-2013 Fonte: elaboração própria com uso do G4Decision e dados do RAIS 2006-2013

Dez estados do país responderam por $85,97 \%$ do número total de postos de trabalho nesta área, no ano de 2013. Embora São Paulo seja o mais representativo (33,7\%), as atividades cresceram principalmente em outras localidades. Ao se tomar como base as taxas médias de crescimento dos dez estados no período de 2006 a 2013, observou-se que São Paulo, Rio de Janeiro e Espirito Santo tiverem crescimento inferior à média nacional, com 6,5\%, 6,1\% e 7\%, respectivamente. Minas Gerais, por sua vez, obteve crescimento médio equivalente à medida nacional $(7,4 \%)$, enquanto Pernambuco $(11,5 \%)$, Goiás $(9,5 \%)$ e Santa Catarina (8,9\%), Paraná (8,6\%), Bahia (8 \%) e Rio Grande do Sul $(7,7 \%)$ a superaram.

Visando identificar os fatores que mais contribuíram para este crescimento, bem como caracterizar estas concentrações de atividades logísticas, foi conduzida uma análise dos três estados com maior taxa de crescimento no período analisado: Pernambuco, Goiás e Santa Catarina.

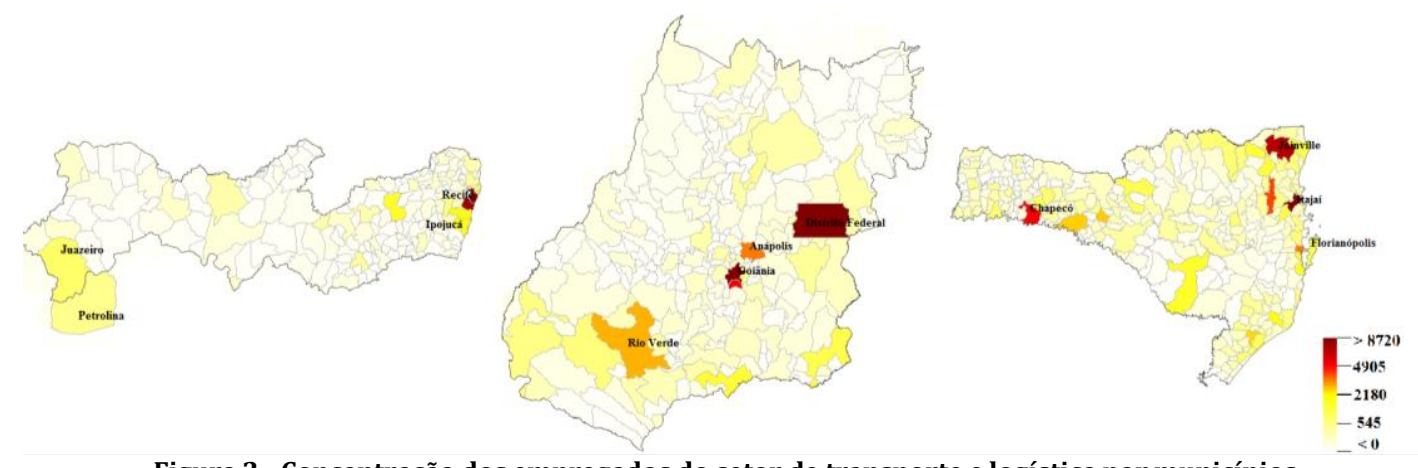

Figura 2 - Concentração dos empregados do setor de transporte e logística por municípios Fonte: elaboração própria com uso do G4Decision e dados do RAIS 2013

\subsection{Os fatores de concentração de atividades logísticas: o estado de Pernambuco}

No estado do Pernambuco, há uma clara concentração de atividades de transporte e logística no litoral, sobretudo na Região Metropolitana de Recife, embora se identifique outras áreas, menos representativas, no interior do estado. Dentre os munícipios com maior número de empregos, relacionados aos grupos de atividades considerados na presente pesquisa, seis localizam-se nos arredores da capital e concentram 78\% do total do Estado. Quatro destes municípios - Recife, Jaboatão dos Guararapes, Olinda e Paulista - tem características semelhantes, em relação aos fatores que explicam a localização de atividades logísticas: i) o acesso ao mercado, pois, conforme IBGE, a região conta com cerca de 3 milhões de habitantes; ii) a presença de importantes eixos de transporte, sobretudo a BR-101 e; iii) a existência do Porto de Recife. Todos estes fatores contribuem para facilitar o acesso a esse mercado e a reduzir os custos de transporte. Há ainda que se considerar, a influência da capital sobre os municípios vizinhos no que se refere aos custos de terrenos: na medida em que os preços dos terrenos aumentam na capital, os municípios localizados no seu entorno passam a ser mais atrativos, devido ao custo inferior - em especial no setor de logística e transporte, cujas instalações demandam bastante espaço e investimentos massivos.

Mais ao sul da capital, em Cabo de Santo Agostinho e Ipojucá, embora ainda próximos dos maiores mercados consumidores do estado e com fácil acesso à infraestrutura de transportes - proximidade da BR-101 e do ramal ferroviário da Companhia Ferroviária do Nordeste - outros fatores justificam a aglomeração das atividades logísticas. 0 Complexo Portuário do Suape constitui o principal polo de investimentos do estado, resultado de um planejamento visando desenvolver o porto e a indústria daquela região. A participação estatal na concepção, financiamento e operação do terminal denota que o fator político e legal exerce grande influência naquela área. Empresas estatais e grandes empresas privadas, a exemplo da Bunge Alimentos e o estaleiro Atlântico Sul, se instalaram no local a partir de 2005, contribuindo para o crescimento de todo o setor de logística e transporte na região.

Além dos municípios já citados, há concentração de atividades logísticas em cidades geograficamente distantes do litoral. É o caso de Petrolina, localizada no extremo oeste do estado, que juntamente com Juazeiro do Norte, no estado vizinho, formam um dos mais importantes polos fruticultores do país. Embora os demais fatores apresentados por Masson e Petiot (2010; 2012) também possam contribuir para esta concentração de atividades logísticas na região, é a necessidade de serviços logísticos que atendam a cadeia de suprimentos da fruticultura que a justifica. Como afirmam Correia, Araújo, e Cavalcanti (2001), concomitantemente à expansão das áreas 
produtivas, ampliou-se naquela região o número de instalações logísticas a fim de atender a demanda em relação ao preparo e conservação dos produtos a serem distribuídos nacional e internacionalmente, como packing houses e armazéns refrigerados.

\subsection{Os fatores de concentração logística: o estado de Goiás}

Em Goiás, podem-se identificar concentrações de atividades logísticas em três principais áreas. Como na maioria dos estados brasileiros, a capital (Goiânia) destaca-se, em função da concentração de atividades econômicas diversas e de sua população. E, de forma semelhante ao que ocorre em vários outros estados, as atividades de transporte e logística aglomeram-se nos municípios vizinhos à capital - como Aparecida de Goiás - devido principalmente à disponibilidade e preços dos terrenos para implantação de novas instalações. Dentre os fatores apresentados por Masson Petiot (2010; 2012), destacam-se, nesta região, o acesso ao mercado e o acesso aos grandes eixos rodoviários, como a rodovia Transbrasiliana (BR-153), principal acesso ao estado e à região.

Em Anapólis, pouco mais ao norte, distinguem-se as atividades industriais das cadeias automobilísticas e farmacêutica. Além dos fatores relacionados às cadeias produtivas e da população local (terceira maior no estado), a presença de uma importante infraestrutura logística favorece a concentração de postos de trabalho dentro dos grupos analisados. Recentemente, um aeroporto industrial de cargas e uma plataforma logística multimodal foram implantados na região.

No sul do estado, principalmente no município de Rio Verde, há outra concentração de atividades logísticas, em região considerada o principal polo produtor de soja de Goiás, onde estão importantes empresas do setor, desde cooperativas até grandes grupos multinacionais. Há ainda a presença de outras empresas relacionadas à cadeia da proteína animal, como a indústria frigorífica e de abate de aves, suínos e bovinos.

\subsection{Os fatores de concentração logística: o estado de Santa Catarina}

A concentração de atividades industriais leva à concentração de atividades de prestação de serviços de transporte e logística, como identificado no Nordeste de Santa Catarina (polo metal mecânico); no Sul (indústria de minerais não metálicos) e, por fim, no Oeste (produção de suínos e aves). Essas atividades atendem tanto a indústria, visando apoiar o seu crescimento, quanto o mercado consumidor que se desenvolve no seu entorno. No entanto, o município que mais se destaca pela concentração de atividades logísticas e, ao mesmo tempo, responde pelo maior Produto Interno Bruto do estado é Itajaí. Embora a região não se destaque pela atividade industrial ou pela população, ali se encontra o maior aeroporto de cargas e o mais importante complexo portuário do estado, este último formado por diversos terminais, dentre os quais APM Terminals, Poly Terminais S/A e Portonave S/A, este último, no município de Navegantes, premiado internacionalmente pela sua eficiência operacional na Lloyd's List Global Awards em 2011 e 2013. Itajaí também abriga o primeiro Instituto de Tecnologia em Logística do País, inaugurado em 2014, e cursos de graduação em logística.

Florianópolis é um dos mais importantes polos de consumo e pode-se identificar uma concentração de atividades logísticas no município de São José, vizinho à capital. Esta concentração é influenciada principalmente por três fatores, dentre os apresentados por Masson e Petiot (2010; 2012): o acesso ao mercado, mais baixos custos de terreno e a acesso aos principais corredores de transporte. Além disso, este munícipio também abriga grandes indústrias, as quais ali se instalam em virtude dos menores custos de terreno e maior facilidade de acesso à infraestrutura rodoviária, em especial a BR-101.

\section{Análise e discussão dos resultados}

A localização das instalações, bem como a natureza das atividades logísticas em cada região, pode ser explicada pelos fatores identificados na literatura e, como afirma Rodrigue et al. (2013), cada fator depende diretamente da natureza das atividades econômicas. Assim, por exemplo, nos municípios destacados como grandes polos de consumo há uma maior concentração de atividades logísticas, como nos casos das capitais dos três estados analisados. Com base na tipologia proposta por Savy (2005), estas aglomerações de instalações, voltadas a atender as demandas logísticas dos grandes mercados consumidores caracterizam-se como zonas logísticas. Na maior parte das vezes, não há um planejamento do território com definição de áreas voltadas à realização de atividades logísticas para atender os grandes centros urbanos, o que contribui para uma concentração de atividades logísticas nas proximidades das capitais, como se pode observar em Recife e Jaboatão dos Guararapes, Goiânia e Aparecida de Goiânia e Florianópolis e São José.

Concentrações de atividades logísticas associadas às atividades industriais também foram identificadas. Nestes casos, fatores relacionados à disponibilidade de infraestrutura de transporte, oferta de fatores de produção são preponderantes. É o caso do município de Anápolis e, especialmente, da região nordeste de Santa Catarina onde, a presença de rodovias, terminais multimodais e portos garante tanto o suprimento para esta indústria quanto o escoamento da produção para os principais polos consumidores do país e do exterior. Esta região continua sendo a localização escolhida para a instalação de novas indústrias, como é o caso da primeira fábrica da BMW no Brasil inaugurada em 2014, no município de Araquari, vizinho a Joinville. Juntamente com estas indústrias se instalam novas empresas prestadoras de serviços logísticos. Nos municípios de Cabo de Santo Agostinho e Ipojucá, em Pernambuco, a natureza das atividades econômicas também é industrial, mas resulta de incentivos governamentais. Concentrações de atividades logísticas são observadas nesta região.

No que se refere à classificação destas concentrações, a região do Nordeste de Santa Catarina, que reúne grandes indústrias e diversos terminais portuários do estado, formam uma grande área logística, segundo a classificação de Savy (2005) como se pode observar na figura 1. A presença de portos e terminais portuários constitui importante fator para a aglomeração de atividades logísticas, tanto que os municípios de Itajaí e Navegantes se destacam pelo número de empresas de transporte e armazenagem e prestadores de serviços auxiliares, como despachantes aduaneiros e agenciadores de cargas. A concentração dessas atividades permite que, nesta região, obtenham-se ganhos relacionados à especialização da mão de obra, resultado do compartilhamento de competências logísticas.

Na região do Suape, pode-se identificar um agrupamento formal e planejado de instalações logísticas, o que caracteriza uma plataforma logística, de acordo com Savy (2005). Por outro lado, as concentrações de atividades logísticas próximas às regiões que se destacam pelas atividades primárias, como agricultura e criação de animais - Vale do Rio São Francisco no oeste do Pernambuco, a região oeste de Santa Catarina e municípios de Rio Verde em Goiás - caracterizam exemplos de zonas logísticas, onde a aglomeração de estabelecimentos resulta da conjunção de fatores que levam as empresas, individualmente, a se localizarem numa mesma região. 


\section{Conclusão}

O presente estudo permitiu verificar o fenômeno de concentração de atividades logísticas no Brasil, o qual foi identificado com base em informações relativas ao número de empregados no setor de logística e transporte. Os fatores que influenciam a decisão de localização destas instalações, bem como aqueles que promovem a concentração das mesmas foram identificados em três estados. Observou-se que diferentes fatores levam à formação de zonas, plataformas ou polos logísticos, os quais tem, claramente, vocações distintas - que se refletem nos diferentes serviços logísticos oferecidos em cada uma destas concentrações. Como exemplo, as regiões próximas aos terminais portuários destacam-se pela aglomeração de atividades de transporte, armazenagem e de serviços auxiliares, como despachantes aduaneiros e agenciadores de cargas. Na literatura, os trabalhos acerca desse tema estão, geralmente, limitados ao estudo das plataformas, mas não tratam de concentrações não planejadas de atividades logísticas, as chamadas zonas logísticas na classificação de Savy (2005). Vale ressaltar, no entanto, que estas últimas são muito mais frequentes tanto que as únicas concentrações planejadas identificadas foram o Complexo Portuário de Suape e a Plataforma Multimodal de Anápolis.

A aglomeração de atividades logísticas tem um impacto positivo, principalmente quando se leva em conta o aspecto de desenvolvimento de uma região ou a possibilidade de obtenção de compartilhamento de recursos mas, quando realizada desordenadamente, pode gerar inúmeras externalidades negativas. A localização das atividades logísticas, como destaca Masson e Petiot (2010; 2012), constituem a manifestação espacial da organização dos fluxos e seu impacto sobre a infraestrutura de transportes e a organização do território deve ser considerado em estudos sobre este tema. Por fim, pode-se destacar que o método usado neste trabalho, para identificar as concentrações de atividades logísticas e a taxa de crescimento destas atividades entre 2006 e 2013, considera somente o número de funcionários das empresas cujas atividades econômicas estão relacionadas diretamente à prestação de serviços de transporte e logística.

Agradecimentos

À Coordenação de Aperfeiçoamento de Pessoal de Nível Superior (CAPES) pelo apoio financeiro.

\section{Referências}

Afandizadeh, S. ., \& Moayedfar, R. (2008). The feasibility study on creation of freight village in Hormozgan Province. Transport, 23(2), 167171.

Ballou, R. H. (2001). Gerenciamento da cadeia de suprimentos: planejamento, organização e logística empresarial. Bookman.

Cambra-Fierro, J., \& Ruiz-Benitez, R. (2009). Advantages of intermodal logistics platforms: Insights from a Spanish platform. Supply Chain Management, 14(6), 418-421.

Correia, R. C., Araújo, J. L. P., \& Cavalcanti, E. de B. (2001). A fruticultura como vetor de desenvolvimento: o caso dos municípios de Petrolina (PE) e Juazeiro (BA). In Congresso brasileiro de economia e sociologia rural (Vol. 39).

Grundey, D., \& Rimienè, K. (2007). Logistics centre concept through evolution and definition. Engineering Economics, 54(4), 87-95.

Higgins, C. ., Ferguson, M. ., \& Kanaroglou, P. (2012). Varieties of logistics centers. Transportation Research Record, (2288), 9-18.

Jařemskis, A. ., \& Vasiliauskas, A. V. . (2007). Research on dry port concept as intermodal node. Transport, 22(3), $207-213$.

Jaržemskis, A. (2007). Research on public logistics centre as tool for cooperation. Transport, 22(1), 50-54.

Jurásková, K., \& Macurová, P. (2013). The study of logistic parks in the Czech republic. Journal of Applied Economic Sciences, 8(3), 299-310.

Krzyzaniak, S., Hajdul, M., \& Fechner, I. (2012). The Concept of a logistics centre model as a nodal point of a transport and logistics network. Archives of Transport, 24(2), 165-186.

$\mathrm{Li}, \mathrm{X}$. (2011). The analysis on the factors of developing zhengzhou city into a regional center of international logistics. Communications in Computer and Information Science, 210 CCIS(PART 3), 586-591.

Masson, S., \& Petiot, R. (2010). Concentration spatiale et activités logistiques. In 8èmes Rencontres Internationales de la Recherche en Logistique. Bordeaux, França.

Masson, S., \& Petiot, R. (2012). Attractivité territoriale, infrastructures logistiques et développement durable. Les Cahiers Scientifiques Du Transport, (61), 63-90.

Meidutè, I. (2005). Comparative analysis of the definitions of logistics centres. Logistikos Centrų Apibrèžimų Lyginamoji Analizè., 20(3), $106-110$.

Rodrigue, J.-P. ., \& Notteboom, T. . (2009). The terminalization of supply chains: Reassessing the role of terminals in port/hinterland logistical relationships. Maritime Policy and Management, 36(2), 165-183.

Rodrigue, J.-P., Comtois, C., \& Slack, B. (2013). The geography of transport systems. Routledge.

Savy, M. (2005). Les plates-formes logistiques. Logistiques Magazine.

Silva, R. M., Senna, E. T. P., Senna, L. A. dos S., \& Júnior, O. F. L. (2013). Governança em plataformas logísticas: uma análise dos elementos e atributos a serem considerados neste tipo de empreendimento logístico. Journal of Transport Literature, 7(3), 240-269.

Silva, R. M., Senna, E. T. P., Senna, L. A. dos S., \& Júnior, O. F. L. (2014). Plataformas Logísticas: uma abordagem sobre as tipologias e características através de uma revisão sistemática. Journal of Transport Literature, 8(1), 210-234.

Tambi, A. M. A., Mohid, M. N., Shukor, I. A., \& Arip, M. S. M. (2013). Planning for a logistics village. World Applied Sciences Journal, 25(3), 421-427.

\section{Abstract}

This paper is about the location and concentration of logistics activities in Brazil, especially in Pernambuco, Goiás and Santa Catarina states. In order to identify these concentrations, data concerning the number of employees who work in economic activities sectors related to transport and logistics were used. The findings indicate concentrations of logistics activities around the large consumer markets, industrial complexes, agricultural production and port terminal areas. Based on the literature, factors contributing to the location decision as well as concentration phenomenon of these facilities are also presented. Lastly, logistic activities concentrations are classified under the taxonomy proposed by Savy (2005).

key words: logistics facilities, lLogistics zones, logistics centers, location decision. 